\title{
David LaGuardia, Gary Ferguson, a cura di, Narrative worlds: essays on the 'nouvelle' in fifteenth and sixteenth century France
}

\section{Filippo Fonio}

\section{(2) OpenEdition}

\section{Journals}

\section{Edizione digitale}

URL: https://journals.openedition.org/studifrancesi/26062

DOI: $10.4000 /$ studifrancesi.26062

ISSN: 2421-5856

\section{Editore}

Rosenberg \& Sellier

\section{Edizione cartacea}

Data di pubblicazione: 1 avril 2007

Paginazione: 159-160

ISSN: 0039-2944

\section{Notizia bibliografica digitale}

Filippo Fonio, «David LaGuardia, Gary Ferguson, a cura di, Narrative worlds: essays on the 'nouvelle' in fifteenth and sixteenth century France», Studi Francesi [Online], 151 (LI | I) | 2007, online dal 30 novembre 2015, consultato il 23 novembre 2021. URL: http://journals.openedition.org/studifrancesi/26062 ; DOI: https://doi.org/10.4000/studifrancesi.26062

Questo documento è stato generato automaticamente il 23 novembre 2021.

\section{cc) (†)}

Studi Francesi è distribuita con Licenza Creative Commons Attribuzione - Non commerciale - Non opere derivate 4.0 Internazionale. 


\title{
David LaGuardia, Gary Ferguson, a cura di, Narrative worlds: essays on the 'nouvelle' in fifteenth and sixteenth century France
}

\author{
Filippo Fonio
}

\section{NOTIZIA}

DAVID LAGUARDIA, GARY FERGUSON, a cura di, Narrative worlds: essays on the 'nouvelle' in fifteenth and sixteenth century France, ACMRS (Arizona Center for Medieval and Renaissance Studies), MRTS («Medieval and Renaissance Texts and Studies», 285), 2005, pp. 195.

1 Il volume esplora alcuni momenti della narrativa breve dei secoli XVI e XVII, insistendo sulla componente finzionale della novella e sulla corrispondente funzione a livello di rispecchiamento e di creazione di un immaginario. Una lettura di questo tipo costituisce un tentativo di oltrepassare la concezione tradizionale della novella come avatar del realismo, e si avvale degli strumenti di critica ed ermeneutica poststrutturalista. Considerare infatti la novella come una pseudomimesi, una tecnica di rappresentazione stereotipa della realtà, non è che un altro modo di valorizzarne la componente oggettiva o presunta tale. Dalla fine del xIX secolo, i primi studi sulla novella si concentrano sullo stabilirne le differenze rispetto agli altri generi della narrativa breve medievale e rinascimentale, e sulla ricerca delle origini dei motivi novellistici. Una costante della letteratura sull'argomento è la considerazione della novella come genere profondamente ibrido, dai cui caratteri conseguono le incertezze nell'identificazione delle fonti, laddove le si cerchi. Vi si possono in particolare identificare di volta in volta tanto fonti narrative, quanto non narrative (Gary FERGUSON, David LAGUARDIA, Introduction, pp. 1-16). L'idea della novella che emerge dai saggi qui 
raccolti è quella di un genere non-mimetico, autoconsapevole, intertestuale, ironico, scettico. La tensione fra reale e finzionale si presenta dunque come cifra per interpretare la novella francese dalla metà del Quattrocento a quella del Cinquecento.

Emily tHOMPSON ("Une merveilleuse espece d'animal": Fable and Verisimilitude in Bonaventure des Périers's Nouvelles récréations et joyeux devis, pp. 17-33) in particolare illustra la componente antirealistica delle novelle di Bonaventure Des Périers, mostrando le interferenze che un genere non-realistico come quello della favola con animali parlanti ingenera. Questo fenomeno richiede al patto finzionale fra autore e lettore una deroga assimilabile alla sospensione dell'incredulità todoroviana. La studiosa considera in particolare le novelle 29 e 87-88 della raccolta di Des Périers, confrontandole con altre opere di finzione a narratore intradiegetico. Anche Tom CONLEY si sofferma sulle Nouvelles récréations di Des Périers, oltre che sui suoi Discours non plus melancoliques que divers (Des Périers on Speed, pp. 35-58), analizzando la maniera in cui le fonti delle due opere (fabliaux, elementi folklorici, letteratura testamentaria, umanesimo, storia recente, vita quotidiana) agiscono nella creazione di una sorta di mappa linguisticoculturale della Francia vernacola nazione unita. Des Périers si presenta al lettore in qualità di etnologo itinerante, i suoi Discours in particolare accentuano la dimensione spaziale della ricerca, all'insegna della velocità (brevitas, pragmatismo, morale laica, colportage). La diffusione attraverso la stampa ha una sua importanza nei confronti di questa enfasi sulla rapida circolazione delle storie e della lingua.

3 I Propos rustiques di Noël Du Fail vengono invece presi in considerazione dal punto di vista del loro rapporto parodico rispetto all'opera di Rabelais (Richard L. REGosIN, Monkey Business: Imitation and the Status of the Text in Du Fail's Propos rustiques, pp. 59-75). Il pesante giudizio di Etienne Pasquier, che considera Du Fail un pessimo imitatore di Rabelais, ha indubbiamente pesato sulla fortuna dei Propos rustiques. Lo studioso considera in questo caso l'inscindibilità del rapporto fra parodia e intentio, in base al quale la dinamica parodica sarebbe individuabile come una intentio dissimulata. L'assenza di una qualsiasi matrice imitativa (intesa come "flattery" e "mockery") sarebbe impensabile, specie per la cultura rinascimentale. Applicando una metodologia decostruzionista, Regosin riconosce già nel termine propos l'enunciazione liminare di una pluralità di intenti (simulatori e dissimulatori) da parte di Du Fail/Maistre Leon Ladulfi.

4 Seguono una serie di studi gender-oriented (Floyd GRAY, Jeanne Flore and Erotic Desire: Feminism or Male Fantasy?, pp. 77-95; Gary FERGUSON, History or Her Story? (Homo)sociality/ sexuality in Marguerite de Navarre's Heptaméron 12, pp. 97-122; John o'BRIEN, Fictions of the Eyewitness, pp. 123-138; David LAGUARDIA, Exemplarity as Misogyny: Variations on the Tale of the One-Eyed Cuckold, pp. 139-158), dedicati a Jeanne Flore e a Marguerite de Navarre. GRAY si interroga sull'identità dell'autore dei Comptes amoureux, considerandone la matrice femminista (in caso si tratti di opera femminile) oppure parodica e misogina (se a scrivere fosse invece un uomo, come lo studioso sostiene). Sono presi in considerazione la dottrina del fol'amor attraverso cui le storie riprese da Ovidio, Virgilio, Jean Lemaire de Belges vengono rilette, il blason come tecnica di rappresentazione e la componente erudita delle novelle, in particolare la riscrittura della fabula di Eco e Narciso. FERGUSON analizza invece una delle prime versioni dell'uccisione di Alessandro de' Medici a opera del cugino Lorenzo, paragonando la dodicesima novella dell'Heptaméron alle cronache del tempo. Marguerite de Navarre in particolare ridimensionerebbe il ruolo del movente politico alla base del gesto di 
Lorenzaccio, per raccontare una storia di difesa dell'onore familiare fino alle estreme conseguenze, da cui non sarebbe assente una rappresentazione delle dinamiche tra gender, potere e autorità, individuabili anche grazie a uno studio degli elementi antropologici e simbolici della novella. O'BRIEN considera alcune novelle di vedovanza dall'Heptaméron, fra possibilità di una riconquista della libertà da parte della vedova $\mathrm{e}$ tentativi di controllo maschili ed ecclesiastici. LAGUARDIA propone invece uno studio tematico, considerando l'evoluzione della storia del marito guercio tradito, dalla Disciplina clericalis alle Cent nouvelles nouvelles, all'Heptaméron. La tradizione misogina da cui deriva questo motivo sarebbe privata di fondamento da parte di Marguerite de Navarre, attraverso una messa in discussione della validità degli exempla e della morale da essi veicolata.

5 Infine Deborah N. LOSSE (Jacques Yver's Le Printemps d'Yver and Trans-Gender Phantasmagoria, pp. 159-172) considera la cifra dell'immaginazione, del 'fantasma', come criterio tematico e strutturante della raccolta di Jacques Yver. La fuga dal realismo in questa raccolta degli anni delle guerre di religione può leggersi come un tentativo di fuga dalla realtà attraverso la fiction (in maniera analoga a quanto avviene, entro certi limiti, nel Decameron e nell'Heptaméron). 